\title{
DEFORMATION MONITORING OF URBAN INFRASTRUCTURE BY TOMOGRAPHIC SAR USING MULTI-VIEW TERRASAR-X DATA STACKS
}

\author{
Sina Montazeri ${ }^{(1)}$, Xiao Xiang Zhu ${ }^{(1,2)}$, Michael Eineder ${ }^{(1,2)}$, Ramon F. Hanssen ${ }^{(3)}$, $\operatorname{Richard~Bamler~}^{(1,2)}$ \\ (1) Remote Sensing Technology Institute (IMF), German Aerospace Center (DLR), Münchner straße 20, \\ Oberpfaffenhofen, 82234 Weßling, Germany, \\ (2) Technische Universität München (TUM), Arcisstraße 21, 80333 Munich, Germany \\ (3) Department of Geoscience and Remote Sensing (GRS), Delft University of Technology, Stevinweg 1, 2628 CN \\ Delft, the Netherlands \\ Email: sina.montazeri@dlr.de,xiao.zhu@dlr.de
}

\begin{abstract}
Synthetic Aperture Radar Tomography (TomoSAR) coupled with data from modern SAR sensors, such as the German TerraSAR-X (TS-X) produces the most detailed three-dimensional (3D) maps by distinguishing among multiple scatterers within a resolution cell. Furthermore, multi-temporal TomoSAR allows for recording the underlying deformation phenomenon of each individual scatterer. One of the limitations of using InSAR techniques, including TomoSAR, is that they only measure deformation along the radar Line-of-Sight (LOS). In order to enhance the understanding of deformation, a decomposition of the observed LOS displacement into the 3D deformation vector in the local coordinate system is desired. In this paper we propose a method, based on L1 norm minimization within local spatial cubes, to reconstruct 3D deformation vectors from TomoSAR point clouds available from, at least, three different viewing geometries. The methodology is applied on two pair of cross-heading TS-X spotlight image stacks over the city of Berlin. The linear deformation rate and amplitude of seasonal deformation are decomposed and the results from two individual test sites with remarkable deformation patterns are discussed in details.
\end{abstract}

\section{INTRODUCTION}

Tomographic SAR inversion (TomoSAR) is, by far, the most advanced InSAR technique which provides highly detailed multi-dimensional maps of the earth's surface [1]. It is a multi-baseline extension of conventional InSAR which allows for multiple scatterer discrimination within each resolution cell [1]-[4]. Therefore, it is favoured over similar InSAR approaches, such as Persistent Scatterer Interferometry (PSI), for studying urban areas in which the prevalent occurrences of layover violate the single scatterer assumption that is made in PSI. By multi-temporal analysis of SAR image stacks TomoSAR is capable to estimate the underlying deformation of individual scatterers in the scene [5]. It is well studied that the most important deformation models using X-band data in urban areas are linear rate and amplitude of seasonal deformation which is induced from thermal dilation of buildings.

One of the limitations of InSAR techniques, including TomoSAR, is that they only measure deformation along the radar Line-of-Sight (LOS). In order to enhance the understanding of deformation, a decomposition of the observed LOS displacement into the $3 \mathrm{D}$ deformation vector is desired. The common method to tackle the aforementioned problem is using a combination of deformation observations from an ascending and a descending track where two out of three components can be reconstructed [6]. For retrieving the third component a prior knowledge about the characteristics of the displacement is necessary [6], [7]. If LOS observations from more than two suitable geometry configurations are available, then it is possible to reconstruct the $3 \mathrm{D}$ displacement vector in the local coordinate system [8]. However, since the LOS deformation estimates obtained from different viewing geometries do not necessarily originate from the same object, a strategy should be introduced to overcome this problem.

In this paper, we propose a method to reconstruct 3D deformation vectors of urban areas from TomoSAR point clouds available from, at least, three different viewing geometries. Initially, TomoSAR point clouds obtained from multiple viewing geometries are geodetically fused in order to produce an accurate shadow-free point cloud. Then around each scatterer a spatial cube is considered within which the 3D displacement vector of the central point is estimated by L1 norm adjustment. The methodology is applied on four TerraSAR-X (TS-X) very high resolution (VHR) spotlight image stacks over the city of Berlin from which two stacks are acquired from ascending geometries and two from descending geometries. The linear deformation rate and amplitude of seasonal motion are decomposed and the results from two test 
sites with remarkable deformation patterns are discussed in details.

\section{INSAR LOS DEFORMATION}

The deformation measurement of SAR techniques $d_{L O S}$ is the projection of the original $3 \mathrm{D}$ displacement vector $\vec{d}$ with components $d_{e}, d_{n}$ and $d_{u}$ in east, north and up direction, respectively, onto the LOS. Assuming a local incidence angle of $\theta_{\text {inc }}$ and a satellite orbit with heading angle $\alpha_{h}$, we can write [6]:

$$
d_{L O S}=d_{u} \cos \left(\theta_{\text {inc }}\right)-d_{A L D} \sin \left(\theta_{\text {inc }}\right),
$$

Where $d_{A L D}$ includes the projection of $d_{n}$ and $d_{e}$ on the azimuth look direction (ALD), that is perpendicular to the satellite flying direction and therefore is expressed as:

$$
d_{A L D}=d_{e} \cos \left(\alpha_{h}\right)-d_{n} \sin \left(\alpha_{h}\right) .
$$

Fig. 1 depicts the aforementioned projection in 3D. If Eq. 2 is substituted in Eq. 1, the explicit relation between deformation measurement $d_{L O S}$ and the displacement components for a single pixel can be written as:

$$
\begin{gathered}
d_{L O S}=d_{u} \cos \left(\theta_{\text {inc }}\right)-d_{e} \cos \left(\alpha_{h}\right) \sin \left(\theta_{\text {inc }}\right) \\
+d_{n} \sin \left(\alpha_{h}\right) \sin \left(\theta_{\text {inc }}\right)
\end{gathered}
$$

From Eq. 3 it is inferred that in order to be able to solve for the three deformation components, at least, three LOS observations from different acquisition geometries are required. Another issue regarding motion decomposition is the sensitivity of InSAR observations with respect to each component. Considering the near polar orbit of TS-X satellites, for instance, with the heading angle of $190.6^{\circ}$ and an incidence angle of $36.1^{\circ}$, the sensitivity decomposition of LOS deformation is $[0.8,0.58,-0.1] \cdot\left[d_{u}, d_{e}, d_{n}\right]^{T}$. Therefore, it is seen that observations are most sensitive to the deformation in the vertical direction and least sensitive to deformation in the north direction. This fact should not be falsely interpreted as ignoring the deformation component $d_{n}$ in the functional model of the decomposition problem stated in Eq. 3. According to Eq. 2, converting $d_{A L D}$ to $d_{e}$ while ignoring $d_{n}$ results in the bias $\Delta \mathrm{d}_{e}$ in the east-west motion component which is expressed as:

$$
\Delta \mathrm{d}_{e}=d_{n} \cdot \tan \left(\alpha_{h}\right) .
$$

With typical satellite azimuth values of TS-X, Eq. 4 demonstrates that the systematic error in east-west component can reach up to $20 \%$ of the deformation in the north-south direction if $d_{n}$ is omitted.

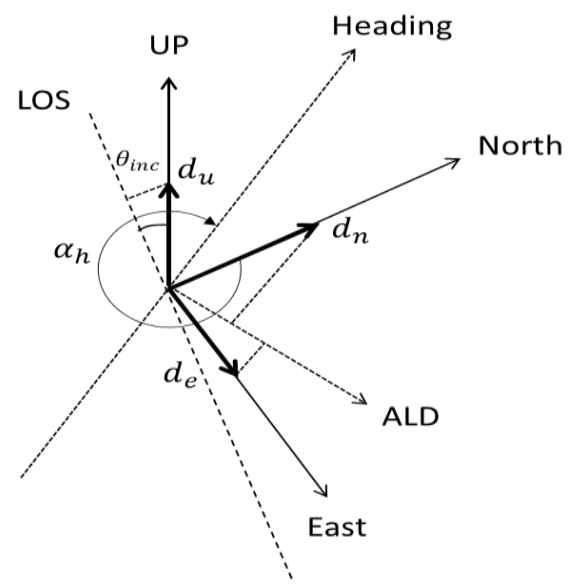

Figure 1. Projection of the original displacement vector $\vec{d}$ with components $\left(d_{e}, d_{n}, d_{u}\right)$ onto the radar $L O S$.

\section{MOTION DECOMPOSITION USING MULTI- ASPECT INSAR OBSERVATIONS}

It is understood from Eq. 3 that availability of LOS observations of the same area from different viewing geometries leads to decomposition of the LOS measurements into their true 3D motion components. It is needless to say that the occurrence of identical scatterers in InSAR, including TomoSAR, point clouds obtained from multiple-viewing geometries is quite rare. Therefore, a strategy is required to estimate the components from available LOS observations. The proposed strategy consists of three steps which are explained in this section.

\subsection{Geodetic point cloud fusion}

Relevant to urban monitoring, the side-looking geometry of SAR satellites only allows capturing the illuminated fraction of buildings. Fusion of point clouds obtained from different same-heading orbits increases the level of details on one side while a combination of results obtained from cross-heading orbits produces shadow-free point clouds. Therefore, merging point clouds obtained from multiple-viewing geometries allows for acquiring the complete shape of individual buildings. The point cloud fusion is carried out based on the selection of an identical reference point during TomoSAR processing of each stack. Beforehand, the exact position of the reference point is calculated by combining more than two SAR measurements, available from different orbits, whose range and azimuth times are corrected for the most prominent error sources, a method called Imaging Geodesy [9] , [10]. Since, the elevation and deformation of scatterers of each stack are evaluated with respect to this point, the point clouds are automatically fused together after geocoding. For more information on geodetic point cloud fusion, the interested reader is referred to [11] and [12]. 


\subsection{Problem formulation within spatial cubes}

The absolutely localized fused point cloud, which is the result of the previous step, is the basis for motion decomposition. However, as it was mentioned earlier, it is unlikely that the same scatterer can be visible from different viewing geometries, especially from crossheading tracks. Therefore, the problem of decomposition is defined in a spatial volume centered on the target point for which the $3 \mathrm{D}$ motion retrieval is desired. The cube is sliding on the points until the entire area of interest is covered. Inside the cube, the necessary information for the follow-on estimation is recorded. Assuming $m$ as the number of points inside the cube, except for the central point, which is constrained to be equal or larger than three in order to guarantee an overdetermined system, and $n$ as number of unknowns, which is equal to three, the observation vector $\mathbf{b}$ is of size $m \times 1$ including either LOS linear rates or LOS amplitudes of seasonal motion attributed to the corresponding scatterers, $\mathbf{X}$ is an $n \times 1$ vector consisting of the three motion components $\left[d_{u}, d_{e}, d_{n}\right]^{T}$ of the central point, $\mathbf{A}$ is the design matrix of size $m \times n$ which is evaluated based on the heading angle of the satellite and the unique local incidence angle relevant to each scatterer (see Eq. 3), and $\mathbf{W}$ is the weight matrix of deformation observations of size $m \times m$. It is important to note that $\mathbf{W}$ is evaluated based on the inverse squared distance of each scatterer relative to the central point i.e. the points closer to the central point have higher weights. After building the required matrices and vectors, we move to the next step which is the estimation of the $3 \mathrm{D}$ motion components of the central point inside the cube.

\subsection{D motion retrieval by $\mathrm{L} 1$ norm adjustment}

Inside each cube centred on the target point, the estimation of motion components is carried out by L1 norm adjustment. With the necessary vector notations already introduced in subsection 3.2, the functional model of our problem is written as:

$$
\mathbf{b}+\mathbf{v}=\mathbf{A} \mathbf{X}
$$

Where $\mathbf{v}$ is the vector of residuals with the same size as the observation vector i.e. $m \times 1$. For the overdetermined system of equations outlined in Eq. 5 $(m \geq n)$, the unknown vector can be retrieved by minimizing the weighted sum of the absolute residuals:

$$
\mathbf{w}^{T}|\mathbf{v}|=\sum_{i=1}^{m} w_{i}\left|v_{i}\right| \rightarrow \min
$$

Where $\mathbf{w}$ is an $m \times 1$ vector which contains the diagonal elements of the weight matrix $\mathbf{W}$. The optimization problem outlined in Eq. 6 is dealt with by linear programming. In this study L1 norm minimization was preferred over the frequently used least squares adjustment due to existence of outliers in the LOS deformation estimates of TomoSAR. It is important to note that L1 norm minimization provides unbiased estimates, like least squares, but the result does not necessarily has minimum variance, unlike least squares adjustment [13].

\section{EXPERIMENTAL RESULTS}

\subsection{Test area and dataset}

In this work, the investigated test site includes the central area of the city of Berlin, Germany. The available dataset consists of four stacks of TS-X VHR spotlight images acquired with a range bandwidth of $300 \mathrm{MHz}$. The images have an azimuth resolution of 1.1 $\mathrm{m}$ and a slant-range resolution of $0.6 \mathrm{~m}$ covering an area of $10 \mathrm{~km} \times 5 \mathrm{~km}$ in a period of six years from March 2008 to March 2013. In terms of viewing geometry, two stacks are acquired from descending orbits with images recorded at 05:20 Coordinated Universal Time (UTC) and two stacks are acquired from ascending tracks with images recorded at 16:50 UTC. Fig. 2 shows the mean scene coverage of individual stacks overlaid on the optical image of Berlin. Furthermore, the details about the system parameters and properties of each stack are summarized in Tab. 1.

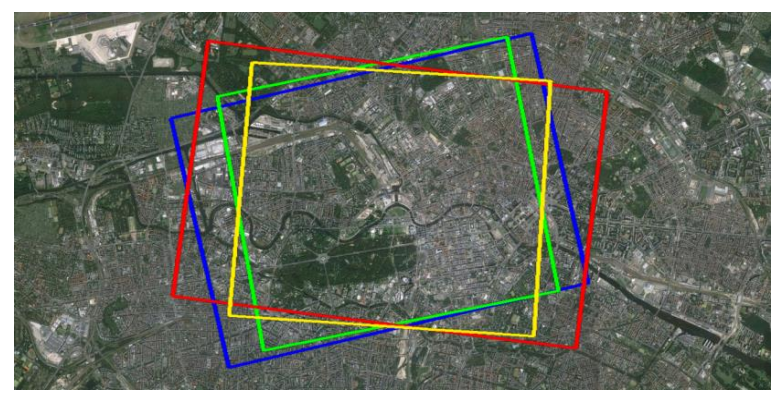

Figure 2. Optical image of the city of Berlin (CGoogle Earth). Rectangles mark the coverage of the four TS-X data stacks.

Table 1. Acquisition parameters of each stack including the average incidence angle, azimuth, the track type and the number of available images.

\begin{tabular}{|c|c|c|c|c|}
\hline Beam & $\begin{array}{c}\text { Incidence } \\
\text { angle }\end{array}$ & $\begin{array}{c}\text { Heading } \\
\text { angle }\end{array}$ & Track type & $\begin{array}{c}\text { Nr. of } \\
\text { Images }\end{array}$ \\
\hline 57 & $41.9^{\circ}$ & $350.3^{\circ}$ & Ascending & 102 \\
\hline 85 & $51.1^{\circ}$ & $352^{\circ}$ & Ascending & 111 \\
\hline 42 & $36.1^{\circ}$ & $190.6^{\circ}$ & Descending & 109 \\
\hline 99 & $54.7^{\circ}$ & $187.2^{\circ}$ & Descending & 138 \\
\hline
\end{tabular}

\subsection{TomoSAR processing and geodetic point cloud fusion}

The InSAR stacking and TomoSAR processing were carried out by the PSI-GENESIS [14] and the TomoGENESIS system [1], [15] of the Remote Sensing 
Technology Institute of DLR. Starting from SLCs, for an input data stack, the Tomo-GENESIS system retrieves the following information: number of layovered scatterers inside each azimuth-range pixel, amplitude and phase, topography and motion parameters (e.g. linear deformation velocity and amplitude of thermal dilation induced seasonal motion) of each detected scatterer. Fig. 3 illustrates the estimated LOS linear deformation rate (a) and amplitude of seasonal motion (b) of beam 42 and beam 57 (c, d), respectively. It can be observed that Berlin is rather stable, i.e. there is no significant ground deformation pattern. Some railway sections, the buildings along them and several buildings under construction undergo a linear subsidence with a rate of up to $8 \mathrm{~mm} / \mathrm{y}$. Most of the buildings and other man-made urban infrastructure mainly undergo temperature change induced seasonal deformation with amplitudes up to $12 \mathrm{~mm}$. The most prominent pattern of seasonal deformation can be seen in the two areas indicated by red ellipses in the seasonal deformation maps of beam 42 and 57. These two areas include the Berlin central railway station and the Eisenbahn bridge. For a more meaningful analysis on the mentioned areas, LOS deformation estimates are decomposed into the motion components (see subsection 4.3). For this purpose TomoSAR point clouds obtained from multiple viewing angles should be correctly fused together. In this experience, since for all of the stacks an identical reference point, whose absolute $3 \mathrm{D}$ position is available, is chosen the multipleview TomoSAR point clouds are geodetically fused. Fig. 4 illustrates the fused TomoSAR point cloud in the UTM (Universal Transverse Mercator) coordinate system. The point cloud covers an area of $10 \mathrm{~km} \times 5 \mathrm{~km}$ and contains approximately 63 million point scatterers. It is observed that fusion of point clouds obtained from cross-heading orbit tracks provide a highly detailed shadow-free $3 \mathrm{D}$ point cloud which is the basis for the motion decomposition.

\subsection{Motion decomposition}

The motion decomposition is done on the two mentioned test cases due to the remarkable LOS seasonal deformation patterns. The LOS linear rate and the amplitude of seasonal deformation are decomposed into the $3 \mathrm{D}$ displacement vector using the methodology explained in Section 3. The size of the cube is chosen to be $5 m \times 5 m \times 5 m$. Fig. 5 shows the TomoSAR LOS seasonal deformation maps of the central station available from each orbit (a-d) as well as the decomposed motion maps in the vertical $(\mathrm{g})$ and the east-west direction (h). In order to justify why L1 norm minimization was preferred over L2 norm, the vertical and east-west motion components evaluated by L2 norm minimization are also shown in (e) and (f). From the LOS maps, seasonal deformation with magnitudes up to $12 \mathrm{~mm}$ is visible. This is mainly due to the construction material of the railway station which consists of steel and glass. LOS maps also show the dependency of the LOS deformation on the acquisition geometry of the satellite as the deformation pattern of ascending and descending tracks is flipped. Fig. 5.g. shows that the main parts of the station and also the bridge in the east of the station undergo vertical seasonal deformation with magnitudes up to $6 \mathrm{~mm}$. This describes $12 \mathrm{~mm}$ of motion between summer and winter due to the expansion and contraction of steel material. The east hallway also shows magnitudes of $5 \mathrm{~mm}$ of vertical seasonal deformation (yellow part). The most interesting pattern is observed in Fig 5.h. The right hallway undergoes heavy seasonal deformation in the east-west direction with magnitudes equal and higher than $12 \mathrm{~mm}$ ( $24 \mathrm{~mm}$ between summer and winter). The railway bridge next to the hallway has smaller magnitudes of motion and moves in the reverse direction with respect to the hallway. The rail tracks located on the west side of the station shows smaller values of horizontal seasonal motion in the order of 5 $\mathrm{mm}$. Another important conclusion is obtained by comparing the estimated components by L1 and L2 norm minimization. This is mainly apparent in Fig.5.e where most of the scatterers are filtered out because of very low precisions. We can conclude that due to the robustness of L1 norm minimization against outliers, this method preserves more information comparing to the results obtained from L2 norm minimization. Furthermore, Due to the insufficient geometry configuration of the available cross-heading tracks, the decomposed motion map in the north-south direction is not reported. However, according to Eq. 4, outlined in Section 2, the component should be kept in the functional model in order to prevent biased estimation of the east-west motion component. Fig. 6 shows the TomoSAR LOS linear deformation maps of the central station available from each orbit (a-d) as well as the decomposed motion maps in the vertical (e) and the east-west direction (f). In general, the building and the rail tracks are not influenced by significant linear deformation as it is observed from the LOS maps. There are some parts in the left side of the station in red colour which shows a construction site and therefore cannot be interpreted as deforming areas. Furthermore, the main building parts of the station show LOS subsidence not higher than $4 \mathrm{~mm} /$ year. The decomposed linear vertical map (see Fig.6.e) shows that the internal sections of the two main parts undergo vertical subsidence in the order of 2 to $4 \mathrm{~mm} /$ year while the rail tracks and other parts of the station are stable.

For the second test site, the Eisenbahn bridge, motion decomposition is only performed on the seasonal deformation map, since there is no significant linear ground deformation visible in the area. In Fig. 7, the seasonal deformation map of Esienbahn railway bridge and its surrounding are analysed. From the seasonal 
LOS maps (see Fig.7.a-d) it can be observed that the two sections on the railway bridge undergo seasonal deformation with magnitudes up to $7 \mathrm{~mm}$. Another prominent pattern can be seen on the building in the top left of each subfigure. From Fig.7.e, it is seen that not much of the LOS seasonal deformation can be attributed to the motion in the vertical direction as for most of the area in the scene magnitudes not higher than $4 \mathrm{~mm}$ are visible. On the other hand, the rail tracks and the building located in the top left are highly influenced by the seasonal deformation in the east-west direction with amplitudes as high as $12 \mathrm{~mm}$. For a detailed interpretation of the deformation pattern on the mentioned building, the reader is referred to [16].

\section{CONCLUSIONS}

In this paper, a methodology was proposed in order to reconstruct the $3 \mathrm{D}$ displacement components from the LOS observations available from tomographic processing of SAR image stacks acquired from multiple viewing geometries. Based on the fused TomoSAR point cloud 3D deformation vector of each scatterer was constructed in a spatial cube by means of L1 norm minimization. The results over Berlin show that manmade objects constructed with steel material undergo seasonal deformation with magnitudes up to $24 \mathrm{~mm}$ between summer and winter. Unfortunately, the geometry configuration of the available image stacks does not allow for reliable retrieval of the deformation in the north-south direction. However the component should be considered in the functional model in order to prevent biased estimation of east-west motion components. Future work concentrates on performing GPS measurements in order to produce absolute deformation maps.

\section{REFERENCES}

1. X. X. Zhu, Very High Resolution Tomographic SAR Inversion for Urban Infrastructure Monitoring: A Sparse and Nonlinear Tour, vol. 666. Deutsche Geodätische Kommission, 2011.

2. A. Reigber and A. Moreira, "First demonstration of airborne SAR tomography using multibaseline L-band data," IEEE Trans. Geosci. Remote Sens., vol. 38, no. 5, pp. 2142-2152, 2000.

3. G. Fornaro, F. Serafino, and F. Soldovieri, "Threedimensional focusing with multipass SAR data," IEEE Trans. Geosci. Remote Sens., vol. 41, no. 3, pp. 507-517, Mar. 2003.

4. X. X. Zhu and R. Bamler, "Very High Resolution Spaceborne SAR Tomography in Urban Environment",
IEEE Trans. Geosci. Remote Sens., vol. 48, no. 12, pp. 4296-4308, 2010.

5. F. Lombardini, "Differential tomography: a new framework for SAR interferometry," IEEE Trans. Geosci. Remote Sens., vol. 43, no. 1, pp. 37-44, Jan. 2005.

6. R. F. Hanssen, Radar Interferometry: Data Interpretation and Error Analysis, Vol. 2. Springer Science \& Business Media, 2001

7. S. Samieie-Esfahany, R. F. Hanssen, K. Thienen-Visser and A. Muntendam-Bos, "On the effect of horizontal deformation on InSAR subsidence estimates", Vol. 30 Proceedings of the Fringe 2009 workshop, Frascati, Italy, 2009.

8. T. J. Wright, B. E. Parsons and L. Zhong, "Toward mapping surface deformation in three dimensions using InSAR", Vol. 31, Geophysical Research Letters, 2004.

9. M. Eineder, C. Minet, P. Steigenberger, X. Cong, and T. Fritz, "Imaging Geodesy - Toward Centimeter-Level Ranging Accuracy With TerraSAR-X," IEEE Trans. Geosci. Remote Sens., vol. 49, no. 2, pp. 661-671, Feb. 2011.

10. X. Cong, U. Balss, M. Eineder, and T. Fritz, "Imaging Geodesy - Centimeter-Level Ranging Accuracy with TerraSAR-X: An Update”, IEEE Geosci. Remote Sens. Lett., vol. 9, no. 5, pp. 948-952, Sep. 2012.

11. S. Montazeri, "The fusion of SAR tomography and Stereo-SAR for 3D absolute scatterer positioning", Delft University of Technology, 2014.

12. X. X. Zhu, S. Montazeri, C. Gisinger, R. F. Hanssen, and R. Bamler, "Geodetic TomoSAR - Fusion of SAR Imaging Geodesy and TomoSAR for 3D Absolute Scatterer Positioning" presented at the Geoscience and Remote Sensing Symposium (IGARSS), 2014 IEEE International, Québec, Canada, 2014.

13. A. R. Amiri-Simkooei, "Formulation of L1 norm minimization in Gauss-Markov model", J. Surv. Eng., Vol. 129, no. 1, pp. 37-43, 2003.

14. N. Adam, B. Kampes, M. Eineder, J. Worawattanamateekul, and M. Kircher, "The development of a scientific permanent scatterer system," in ISPRS Workshop High Resolution Mapping from Space, Hannover, Germany, 2003, vol. 2003, p. 6.

15. X. Zhu, Y. Wang, S. Gernhardt, and R. Bamler, "TomoGENESIS: DLR's Tomographic SAR Processing System," presented at the Urban Remote Sensing Event (JURSE), 2013 Joint, 2013, pp. 159-162.

16. S. Gernhardt and R. Bamler, "Deformation monitoring of single buildings using meter-resolution SAR data in PSI," ISPRS J. Photogramm. Remote Sens., vol. 73, pp. 68-79, Sep. 2012. 


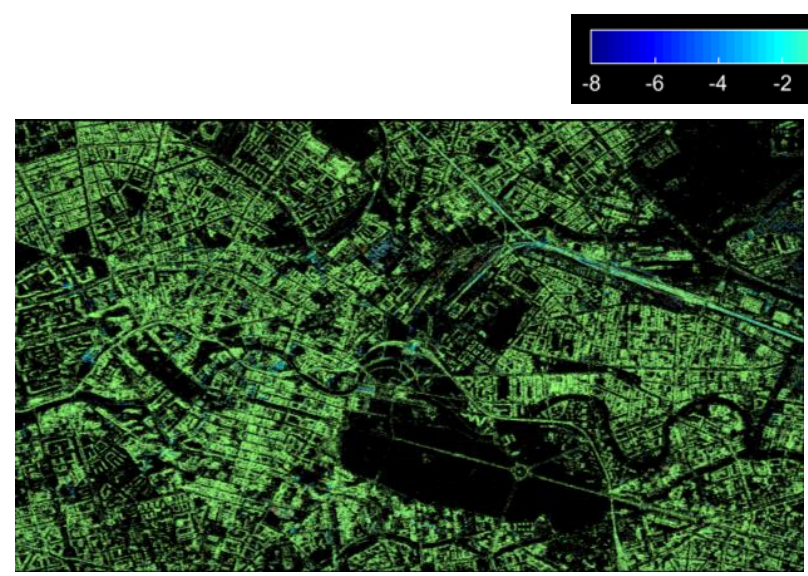

(a) Linear deformation rate $[\mathrm{mm} / \mathrm{y}]$, beam 42

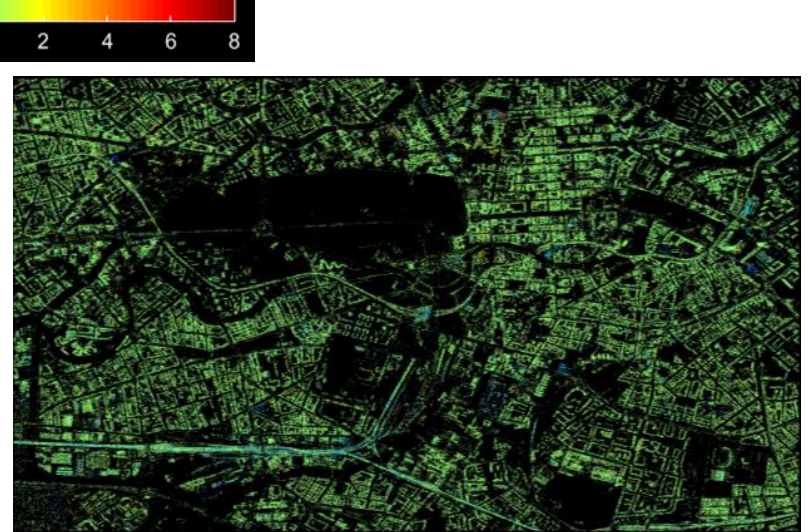

(c) Linear deformation rate $[\mathrm{mm} / \mathrm{y}]$, beam 57

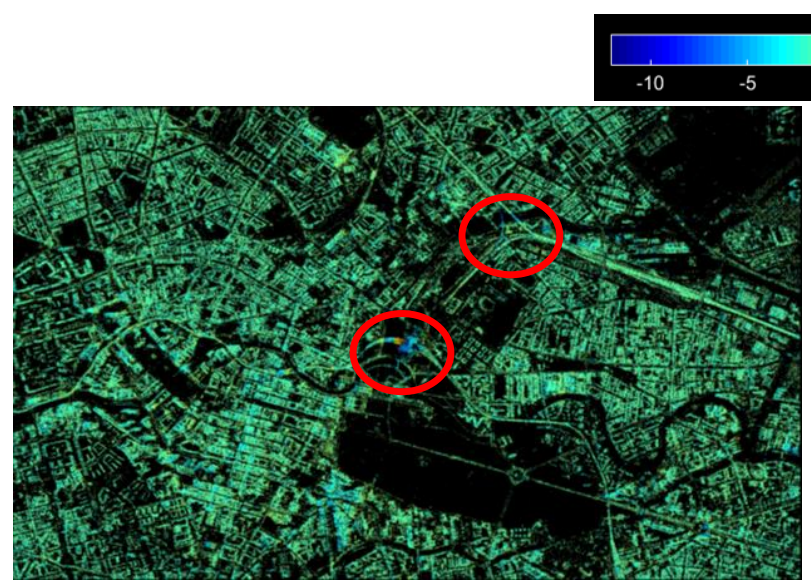

(b) Amplitude of seasonal motion [mm], beam 42

Figure 3. TomoSAR deformation maps. Estimated LOS linear deformation rate (a) and amplitude of seasonal motion (b) of beam 42. Estimated LOS linear deformation rate (c) and amplitude of seasonal motion $(d)$ of beam 57. Motion parameter is color-coded.

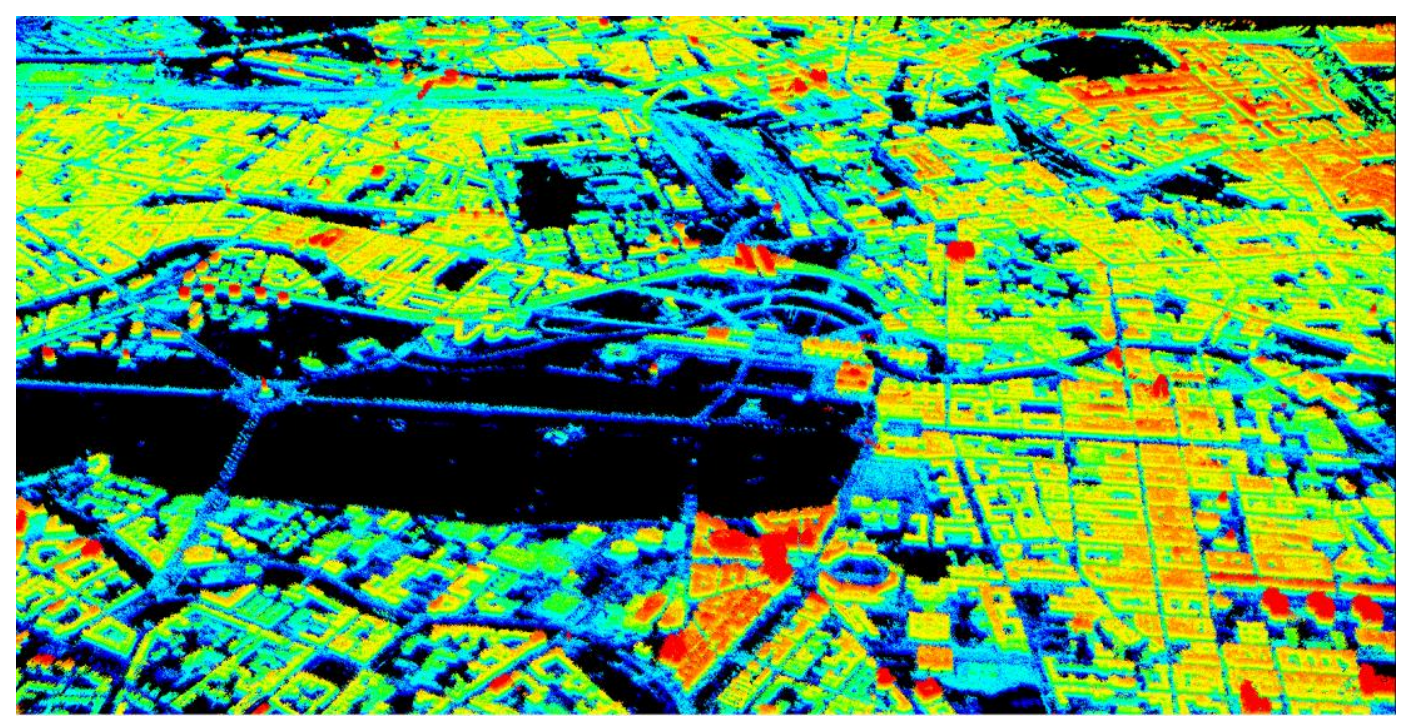

Figure 4. Geodetically fused TomoSAR point cloud of Berlin in 3D. Height is color-coded. 


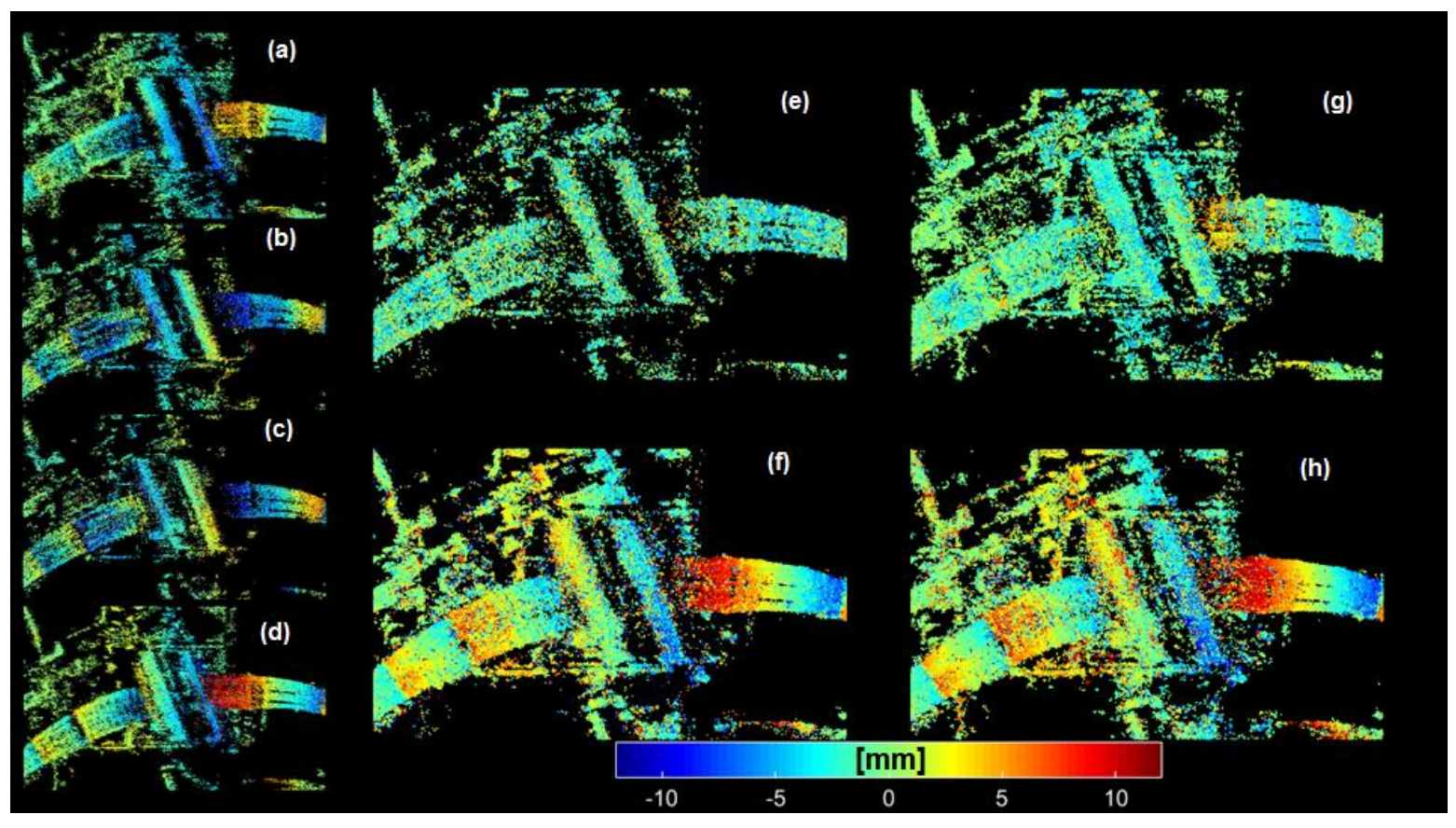

Figure 5. Berlin central railway station. LOS Amplitude of seasonal motion of beam $42(a)$, beam $57(b)$, beam $85(c)$ and beam $99(d)$. Decomposed seasonal deformation in vertical direction (e) and east-west direction $(f)$ by $L 2$ norm minimization. Decomposed seasonal deformation in vertical direction $(g)$ and east-west direction ( $h$ ) by L1 norm minimization. Deformation maps are in $\mathrm{mm}$.

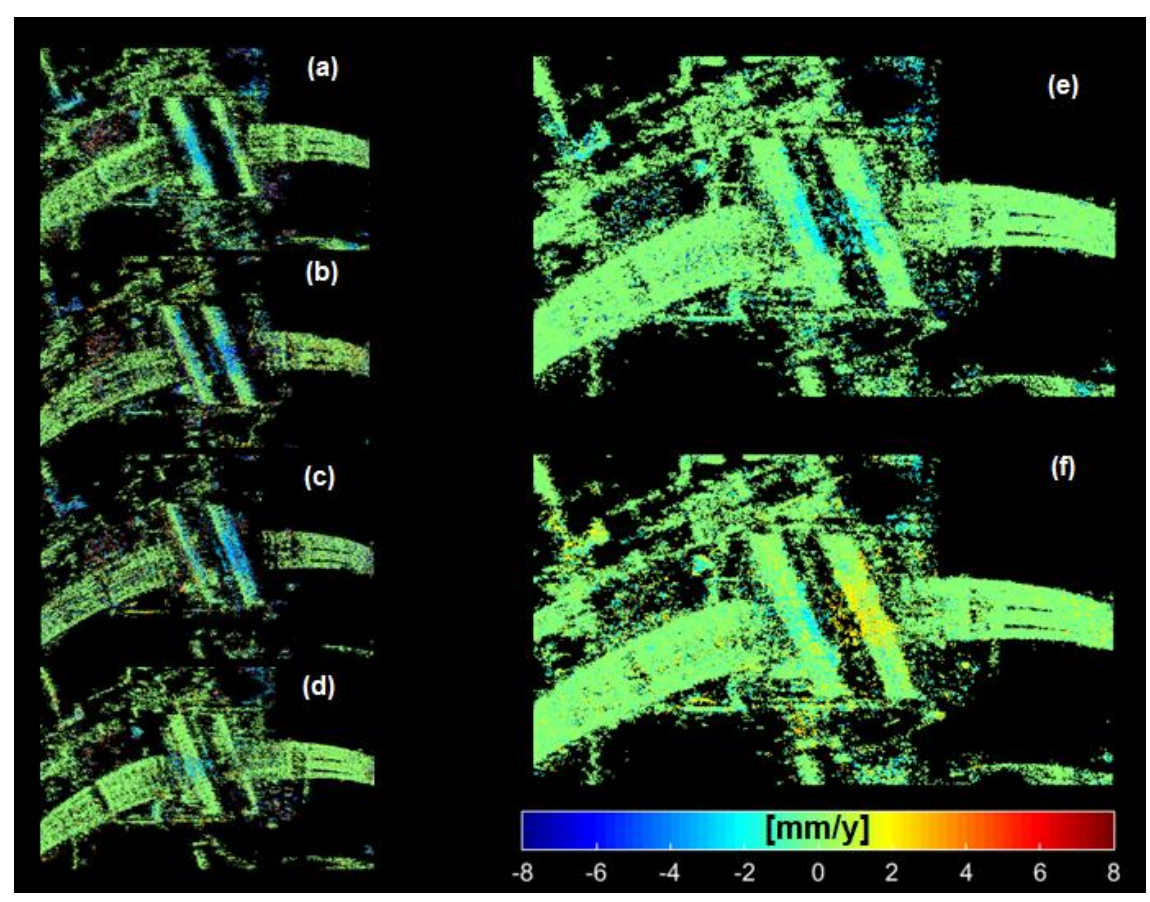

Figure 6. Berlin central railway station. LOS linear motion of beam 42 (a), beam 57 (b), beam 85 (c) and beam $99(d)$. Decomposed linear deformation rate in vertical direction $(e)$ and east-west direction $(f)$.

Deformation maps are in $\mathrm{mm} /$ year. 


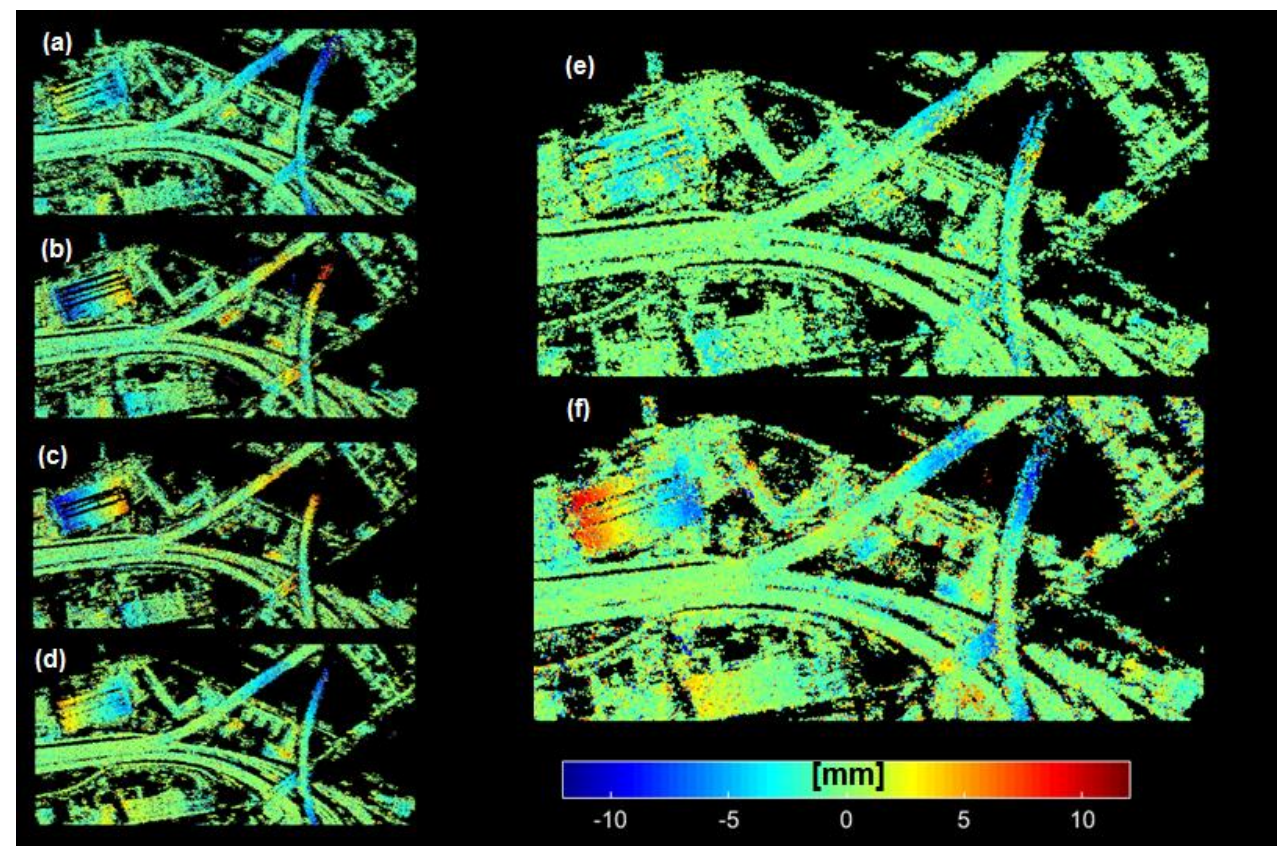

Figure 7. Eisenbahn railway bridge. LOS Amplitude of seasonal motion of beam $42(a)$, beam $57(b)$, beam $85(c)$ and beam $99(d)$. Decomposed seasonal deformation in vertical direction $(e)$ and eastwest direction ( $f$ ) by L1 norm minimization. Deformation maps are in $\mathrm{mm}$. 